\title{
Comparison of concurrent chemoradiotherapy versus sequential radiochemotherapy in patients with completely resected non-small cell lung cancer
}

\author{
Hwan-Ik Kim, MD¹, O Kyu Noh, MD, PhD ${ }^{1,2}$, Young-Taek Oh, MD, PhD¹, Mison Chun, MD, PhD ${ }^{1}$, \\ Sang-Won Kim, MD', Oyeon Cho, MD, PhD'1, Jaesung Heo, MD \\ Departments of ${ }^{1}$ Radiation Oncology and ${ }^{2}$ Biomedical Informatics, Ajou University School of Medicine, Suwon, Korea
}

\begin{abstract}
Purpose: Our institution has implemented two different adjuvant protocols in treating patients with non-small cell lung cancer (NSCLC): chemotherapy followed by concurrent chemoradiotherapy (CT-CCRT) and sequential postoperative radiotherapy (PORT) followed by postoperative chemotherapy (POCT). We aimed to compare the clinical outcomes between the two adjuvant protocols. Materials and Methods: From March 1997 to October 2012, 68 patients were treated with CT-CCRT $(n=25)$ and sequential PORT followed by POCT (RT-CT; $n=43$ ). The CT-CCRT protocol consisted of 2 cycles of cisplatin-based POCT followed by PORT concurrently with 2 cycles of POCT. The RT-CT protocol consisted of PORT followed by 4 cycles of cisplatin-based POCT. PORT was administered using conventional fractionation with a dose of 50.4-60 Gy. We compared the outcomes between the two adjuvant protocols and analyzed the clinical factors affecting survivals.

Results: Median follow-up time was 43.9 months (range, 3.2 to 74.0 months), and the 5-year overall survival (OS), locoregional recurrence-free survival (LRFS), and distant metastasis-free survival (DMFS) were $53.9 \%, 68.2 \%$, and $51.0 \%$, respectively. There were no significant differences in OS ( $p=0.074)$, LRFS $(p=0.094)$, and DMFS $(p=0.490)$ between the two protocols. In multivariable analyses, adjuvant protocol remained as a significant prognostic factor for LRFS, favouring CT-CCRT (hazard ratio [HR] $=3.506, p=$ 0.046) over RT-CT, not for OS (HR $=0.647, p=0.229$ ).

Conclusion: CT-CCRT protocol increased LRFS more than RT-CT protocol in patients with completely resected NSCLC, but not in OS. Further studies are warranted to evaluate the benefit of CCRT strategy compared with sequential strategy.
\end{abstract}

Keywords: Non-small-cell lung carcinoma, Adjuvant chemotherapy, Adjuvant radiotherapy, Sequence of therapies

\section{Introduction}

Because surgery alone is not a satisfactory strategy for the treatment of non-small cell lung cancer (NSCLC), adjuvant treatment with postoperative chemotherapy (POCT) and postoperative radiotherapy (PORT) is usually recommended.
Cisplatin-based POCT is the standard of care for stage IIIII NSCLC patients who undergo complete resection [1,2]. However, the benefit of PORT has been an issue of debate. A meta-analysis showed that while PORT significantly lowered local recurrence, it had a detrimental effect on survival [3]. However, such harmful effect on survival can be attributed

Received 23 May 2016, Revised 1 August 2016, Accepted 12 August 2016.

Correspondence: $\mathrm{O}$ Kyu Noh, MD, PhD, Departments of Radiation Oncology and Biomedical Informatics, Ajou University School of Medicine, 164 Worldcup-ro, Yeongtong-gu, Suwon 16499, Korea. Tel: +82-31-219-5884, Fax: +82-31-219-5894, E-mail: okyu.noh@gmail.com

(C) This is an Open Access article distributed under the terms of the Creative Commons Attribution Non-Commercial License (http://creativecommons.org/ licenses/by-nc/4.0/) which permits unrestricted non-commercial use, distribution, and reproduction in any medium, provided the original work is properly cited.

www.e-roj.org 
to old-fashioned radiotherapy techniques with increased cardiopulmonary toxicity. Modern radiotherapy using linear accelerator can improve local control and survival [4]. A largescaled retrospective study using data set of Surveillance, Epidemiology, and End Results (SEER) reported a survival benefit of PORT for N2 disease [5]. The Adjuvant Navelbine International Trialist Association (ANITA) trial also showed a potential benefit of PORT for N2 disease in the setting of POCT [6]. Recently, a review of the data from the National Cancer Data Base (NCDB) showed additional survival benefit of PORT in patients with $\mathrm{N} 2$ disease who received adjuvant chemotherapy [7-9]. Therefore, PORT can be considered as an adjuvant therapy after POCT in patients with N2 disease as well as those with a close or positive resection margin status.

In October 1996, before POCT became a standard of care for stage II-III NSCLC, adjuvant chemotherapy followed by concurrent chemoradiotherapy (CT-CCRT) was established as an institutional protocol for patients showing pathologic N1-2 disease (mainly N2) or a close/positive resection margin. After POCT became a standard of care, sequential PORT followed by POCT (RT-CT) has been a routine protocol in our institution with a consensus of a multidisciplinary team [10].

In cases where both POCT and PORT are administered after surgery, the optimal way of combining these two therapies has not yet been discovered. In this study, we aimed to compare the clinical outcomes between our two historical protocols (CT-CCRT vs. RT-CT).

\section{Materials and Methods}

Using our institutional tumor registry database, we identified 68 patients who underwent both PORT and POCT after complete surgical resection for NSCLC between 1997 and 2012. For staging work-up, chest computed tomography (CT), bronchoscopy, enhanced brain CT or magnetic resonance imaging $(\mathrm{MRI})$, and bone scan or positron emission tomography (PET) were routinely performed. Lobectomy or pneumonectomy with mediastinal lymph node dissection were performed in patients with clinical N0-1 or singlestation minimal N2 disease. Pathologic stages were described according to the 7th edition of American Joint Committee on Cancer (AJCC) TNM classification.

PORT was administered in patients with pathologic N1-2 disease (mainly N2) and positive or close resection margins. Before 2002, PORT was performed using the conventional twodimensional technique (2D-RT) with megavoltage beams ( $\geq 6$ MV). Initial anterior/posterior-posterior/anterior fields included an ipsilateral hilum and involved lymph nodal stations plus its next draining stations, and a dose of 30.6-41.4 Gy using conventional fractionation (1.8-2.0 Gy/day) was irradiated. Two off-cord oblique fields were implemented to boost the ipsilateral hilum and involved nodal stations with a dose up to 50.4-60.0 Gy. After 2002, three-dimensional conformal radiation therapy (3D-CRT) was adopted using megavoltage photon beam. For 3D-CRT, CT simulation was scanned under the free-breathing condition. The initial clinical target volume (CTV) included a bronchial stump, involved mediastinal lymph nodal stations, and its next draining stations. The boost CTV only included a bronchial stump and involved nodal stations. The planning target volume (PTV) was expanded in all directions from the CTV with a margin of $1.0-1.5 \mathrm{~cm}$. Conventional fractionation was used with a dose of 44-45 Gy for initial volume, and the boost volume was irradiated up to 50.4-60.0 Gy. In case of a close resection margin (less than 5 $\mathrm{mm})$, the region of the close margin was boosted up to doses of $66 \mathrm{~Gy}$.

POCT was administered in patients with pathologic stage II/III according to our institutional protocols; 1) CT-CCRT protocol (adjuvant chemotherapy followed by concurrent chemoradiotherapy): two cycles of cisplatinbasted chemotherapy were administered, followed by PORT concurrently with two cycles of chemotherapy [11] and 2) RTCT protocol (sequential PORT followed by POCT): PORT was administered within 4-6 weeks after surgery, followed by 4 cycles of cisplatin-based adjuvant chemotherapy [10].

To assess comorbidity, we calculated an age-adjusted Charlson comorbidity score by using of previous established International Classification of Disease-10 diagnosis codes from inpatient and outpatient records-from the time of first visit of each patient to the date of surgical resection [12].

We then analyzed the pattern of first failures and clinical parameters influencing locoregional recurrence-free survival (LRFS), distant metastasis-free survival (DMFS) and overall survival (OS). We compared the pattern of first failures and survivals between CT-CCRT and RT-CT protocols. Comparison between the two protocols was analyzed using a chi-square test or Fisher exact test for categorical variables and the Student t-test or the Mann-Whitney $U$ test for continuous variables. Survival time was calculated by the interval between the date of the surgery and the date of the last follow-up or events (death event for OS, first loco-regional failure for LRFS, and first distant metastasis for DMFS). Survivals were calculated using the Kaplan-Meier method. The log-rank test and Cox proportional hazards regression model were used for 
univariable and multivariable analyses, respectively. Factors with a $p$-value of less than 0.2 by a univariable analysis were used for a multivariable analysis. Two sided p-values less than 0.05 were regarded as statistically significant. All statistical analyses were performed using $R$ statistical packages [13]. This study was reviewed and approved by the Ajou University School of Medicine Institutional Review Board (AJIRB-MEDMDB-13-036).

\section{Results}

Among a total of 68 patients, 45 patients (66.2\%) were males and the median age was 58 years (range, 30 to 69 years). The CT-CCRT and RT-CT protocols were administered in 25 (36.8\%) and 43 patients (63.2\%), respectively. The types of surgery included lobectomy in 53 patients (77.9\%) and pneumonectomy in 15 (22.1\%). Squamous cell carcinoma and adenocarcinoma were observed in 36 patients (52.9\%) and 24 patients (35.3\%), respectively. Pathologic nodal stages were N1 in 15 patients (22.1\%) and N2 in 51 patients (75.0\%), while 2 patients with pNO disease showed a close resection margin of less than $5 \mathrm{~mm}$. The median dose of PORT was $54.0 \mathrm{~Gy}$ (range, 39.6 to $64.4 \mathrm{~Gy}$ ) and the median cycle of POCT was 4 (range, 2 to 6).

Patient characteristics for CT-CCRT and RT-CT protocols are summarized in Table 1. There were no significant differences in proportions of gender, history of smoking, comorbidity index, tumor histology, type of surgery, T-stage and N-stage between the two protocols. The age of the patients in the CTCCRT protocol was significantly older than that of patients in the RT-CT protocol (mean age, 58.7 vs. 52.9 years; $p=0.025$ ). The proportion of patients showing ECOG PS 2 in the RT-CT protocol was significantly higher than that of patients in the CT-CCRT protocol (0\% vs. $14.0 \% ; p=0.050$ ). The absolute value of preoperative forced expiratory volume in 1 second (FEV1) in the RT-CT protocol was significantly higher than that of the CT-CCRT protocol (mean FEV1, 2.2 L vs. 2.7 L; $p=0.002$ ). The postoperative FEV1 was available in 35 patients (51.5\%) and there was no significant difference between the two protocols. Twenty-one patients (84.0\%) who underwent the CTCCRT protocol were treated by 2D-RT, while 42 patients (97.7\%) who received the RT-CT protocol were treated with $3 \mathrm{D}-\mathrm{CRT}$. Radiation was delivered with a significantly higher dose in the RT-CT protocol compared to that in the CT-CCRT protocol (mean dose, 52.8 Gy vs. $55.8 \mathrm{~Gy}, \mathrm{p}=0.013$ ). The median POCT cycle was 4 in both protocols. Mild to moderate radiation esophagitis was found in 2 patients (8.0\%) for the CT-CCRT
Table 1. Patient characteristics according to treatment protocols

\begin{tabular}{|c|c|c|c|}
\hline Characteristic & $\begin{array}{l}\text { CT-CCRT } \\
(n=25)\end{array}$ & $\begin{array}{c}\text { RT-CT } \\
(n=43)\end{array}$ & $p$-value \\
\hline Age (yr) & $58.7 \pm 8.4$ & $52.9 \pm 10.7$ & 0.025 \\
\hline Gender & & & 0.106 \\
\hline Male & 13 & 32 & \\
\hline Female & 12 & 11 & \\
\hline Smoking history & & & 0.579 \\
\hline Yes & 10 & 13 & \\
\hline No & 15 & 30 & \\
\hline ECOG PS & & & 0.050 \\
\hline $0-1$ & 25 & 37 & \\
\hline 2 & 0 & 6 & \\
\hline Comorbidity index & & & 0.206 \\
\hline$<3$ & 13 & 29 & \\
\hline$\geq 3$ & 12 & 14 & \\
\hline Preoperative FEV1 (L) & $2.2 \pm 0.5$ & $2.7 \pm 0.7$ & 0.002 \\
\hline Postoperative FEV1 (L) & $2.2 \pm 0.9$ & $2.0 \pm 0.6$ & 0.718 \\
\hline Tumor histology & & & 0.694 \\
\hline Squamous cell & 13 & 23 & \\
\hline Adenocarcinoma & 10 & 14 & \\
\hline Others & 2 & 6 & \\
\hline Type of surgery & & & 0.550 \\
\hline Lobectomy & 18 & 35 & \\
\hline Pneumonectomy & 7 & 8 & \\
\hline Pathologic T stage & & & 0.347 \\
\hline $\mathrm{T} 1$ & 5 & 4 & \\
\hline T2 & 13 & 29 & \\
\hline T3 & 7 & 10 & \\
\hline Pathologic N stage & & & 0.095 \\
\hline No & 2 & 0 & \\
\hline N1 & 7 & 8 & \\
\hline N2 & 16 & 35 & \\
\hline Radiotherapy technique & & & $<0.001$ \\
\hline Two-dimensional & 21 & 1 & \\
\hline Three-dimensional & 4 & 42 & \\
\hline Radiotherapy dose (Gy) & $52.8 \pm 5.5$ & $55.8 \pm 4.3$ & 0.013 \\
\hline
\end{tabular}

Values are presented as mean \pm standard deviation or number. CT-CCRT, adjuvant chemotherapy followed by concomitant chemoradiotherapy; RT-CT, sequential postoperative radiotherapy followed by adjuvant chemotherapy; ECOG PS, Eastern Cooperative Oncology Group performance status; FEV1, forced expiratory volume in 1 second.

a) Postoperative FEV1 was available in 35 patients (51.5\%).

protocol, and in 11 patients (25.6\%) for the RT-CT protocol $(p=0.145)$. The rate of symptomatic radiation pneumonitis treated with steroid was not significantly different between the two protocols (24.0\% vs. 9.3\%, $p=0.195)$. There was also no significant difference in hematologic toxicities of grade $3-4$ (20.0\% vs. $46.5 \%, p=0.054)$. Comparing the pattern of first failures, there were no significant differences in locoregional 
Table 2. Pattern of first failures according to treatment protocols

\begin{tabular}{lccc}
\hline \multicolumn{1}{c}{ First failure site } & CT-CCRT $(n=25)$ & RT-CT $(n=43)$ & p-value \\
\hline Locoregional & $0(0)$ & $2(4.7)$ & 0.528 \\
Distant metastasis & $6(24.0)$ & $7(16.3)$ & 0.527 \\
Both locoregional and distant metastasis & $4(16.0)$ & $13(30.2)$ & 0.251 \\
\hline
\end{tabular}

Values are presented as number (\%).

CT-CCRT, adjuvant chemotherapy followed by concomitant chemoradiotherapy; RT-CT, sequential postoperative radiotherapy followed by adjuvant chemotherapy.

Table 3. Univariable analyses for clinical variables affecting survivals

\begin{tabular}{|c|c|c|c|c|c|c|}
\hline Variable & $5-y r$ LRFS $(\%)$ & $\begin{array}{c}\text { p-value } \\
\text { (log-rank) }\end{array}$ & $5-y r$ DMFS $(\%)$ & $\begin{array}{c}\text { p-value } \\
\text { (log-rank) }\end{array}$ & $5-y r$ OS $(\%)$ & $\begin{array}{c}\text { p-value } \\
(\log \text {-rank) }\end{array}$ \\
\hline Age (<58 vs. $\geq 58$ yr) & 68.6 vs. 67.2 & 0.632 & 50.0 vs. 52.0 & 0.793 & 65.3 vs. 43.3 & 0.072 \\
\hline Gender (male vs. female) & 73.0 vs. 59.4 & 0.071 & 55.3 vs. 42.7 & 0.084 & 53.6 vs. 54.6 & 0.855 \\
\hline Smoking history (no vs. yes) & 65.3 vs. 69.5 & 0.223 & 48.0 vs. 52.0 & 0.233 & 60.8 vs. 50.7 & 0.472 \\
\hline ECOG PS (0-1 vs. 2) & 69.3 vs. 53.3 & 0.188 & 54.7 vs. 0.0 & 0.174 & 53.4 vs. 62.5 & 0.955 \\
\hline Comorbidity index (<2 vs. $\geq 2$ ) & 62.8 vs. 70.4 & 0.873 & 51.5 vs. 50.6 & 0.861 & 66.2 vs. 49.4 & 0.081 \\
\hline Preoperative FEV1 (<2.5 L vs. $\geq 2.5$ L) & 64.3 vs. 73.8 & 0.189 & 47.5 vs. 57.5 & 0.407 & 46.7 vs. 61.9 & 0.378 \\
\hline Surgery (lobectomy vs. pneumonectomy) & 65.3 vs. 82.5 & 0.375 & 49.5 vs. 54.5 & 0.961 & 56.0 vs. 45.7 & 0.242 \\
\hline Tumor histology (squamous vs. others) & 63.7 vs. 74.1 & 0.297 & 42.7 vs. 61.5 & 0.165 & 55.3 vs. 51.6 & 0.786 \\
\hline Pathologic T stage (1-2 vs. 3) & 69.4 vs. 63.5 & 0.747 & 54.8 vs. 41.7 & 0.612 & 55.9 vs. 49.3 & 0.573 \\
\hline Pathologic N stage (0-1 vs. 2) & 67.0 vs. 68.7 & 0.424 & 44.7 vs. 52.8 & 0.835 & 50.4 vs. 55.2 & 0.999 \\
\hline Radiation dose (<54 Gy vs. $\geq 54$ Gy) & 72.2 vs. 64.4 & 0.548 & 59.0 vs. 44.9 & 0.675 & 51.2 vs. 56.1 & 0.885 \\
\hline Protocol (CT-CCRT vs. RT-CT) & 82.8 vs. 60.3 & 0.094 & 59.9 vs. 46.4 & 0.490 & 44.0 vs. 61.3 & 0.074 \\
\hline
\end{tabular}

LRFS, locoregional recurrence-free survival; DMFS, distant metastasis-free survival; OS, overall survival; ECOG PS, Eastern Cooperative Oncology Group performance status; FEV1, forced expiratory volume in 1 second; CT-CCRT, adjuvant chemotherapy followed by concomitant chemoradiotherapy; RT-CT, sequential postoperative radiotherapy followed by adjuvant chemotherapy.

recurrence $(L R)$, distant metastasis (DM), and both $L R$ and $D M$ between the two protocols (Table 2).

The median follow-up time of 68 patients was 43.9 months (range, 3.2 to 74.0 months), and the $0 \mathrm{~S}$ was $53.9 \%$ at 5 years. The 5-year LRFS and DMFS were $68.2 \%$ and $51.0 \%$, respectively. Patients with CT-CCRT protocol showed favourable LRFS, but unfavourable OS compared to the RT-CT protocol (5-year LRFS, $82.8 \%$ vs. $60.3 \%, p=0.094 ; 5$-year $0 S, 44.0 \%$ vs. $61.3 \%, p=$ $0.074)$. There was no significant difference in DMFS between the two protocols (5-year DMFS, $53.3 \%$ vs. $46.4 \%, p=0.490$ ) (Table 3).

Univariable analyses for clinical variables influencing survival are presented in Table 3. There was no significant factor affecting OS, LRFS, and DMFS. The age at diagnosis ( $<58$ vs. $\geq 58$ ) and comorbidity index ( $<2$ vs. $\geq 2$ ) were found to be marginally significant factors affecting OS (age, $p=$ 0.072 ; comorbidity index, $p=0.081$ ). Male patients showed favourable trend compared to female patients in LRFS ( $p=$ $0.071)$ and DMFS ( $p=0.084)$.

The results of multivariable analysis are summarized in Table
4. There were no significant variables affecting on DMFS and OS (Fig. 1A and 1C). The adjuvant protocol (CT-CCRT vs. RT$\mathrm{CT}$ ) remained as a statistically significant prognostic factor for LRFS (HR = 3.506, $p=0.046)$ (Fig. 1B).

\section{Discussion and Conclusion}

In this study, we compared the outcomes of two institutionally historical protocols (CT-CCRT vs. RT-CT) in patients with completely resected NSCLC, and our results showed that the CT-CCRT protocol had statistically significant favourable prognostic value in LRFS compared with the RT-CT protocol, while there were no differences in OS or DMFS. Patient characteristics were not different between two protocols except age, ECOG PS, preoperative FEV1, radiotherapy technique and radiation dose (Table 1). Although preoperative FEV1 was significantly different (mean, 2.2 L vs. 2.7 L, $p=0.002$ ), postoperative FEV1, which could have a significant effect on OS [14], was not different between the two protocols. The higher proportion of ECOG PS 2 may have influenced the poorer 
A

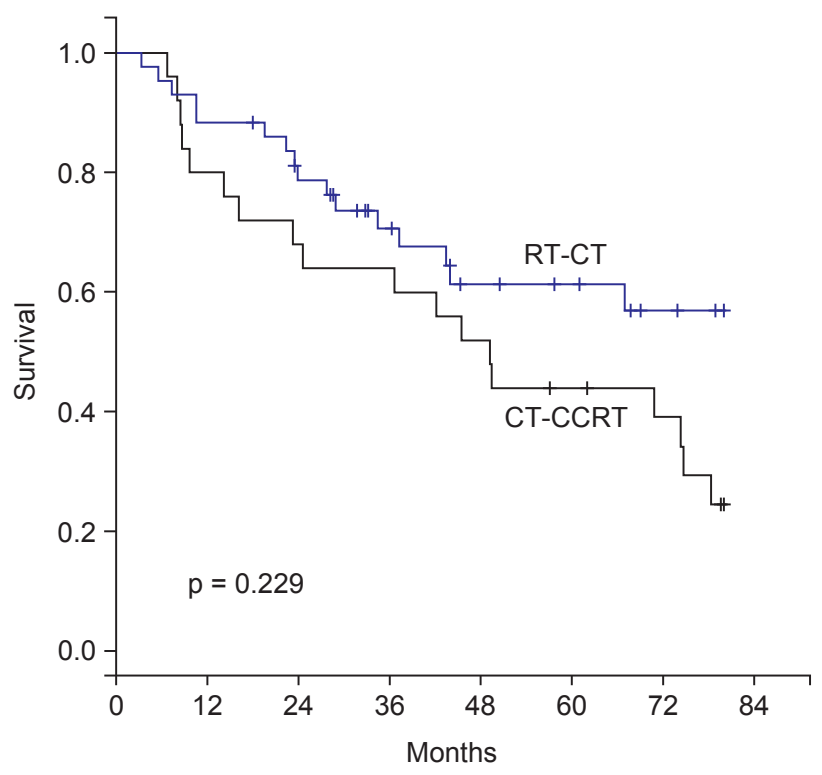

C

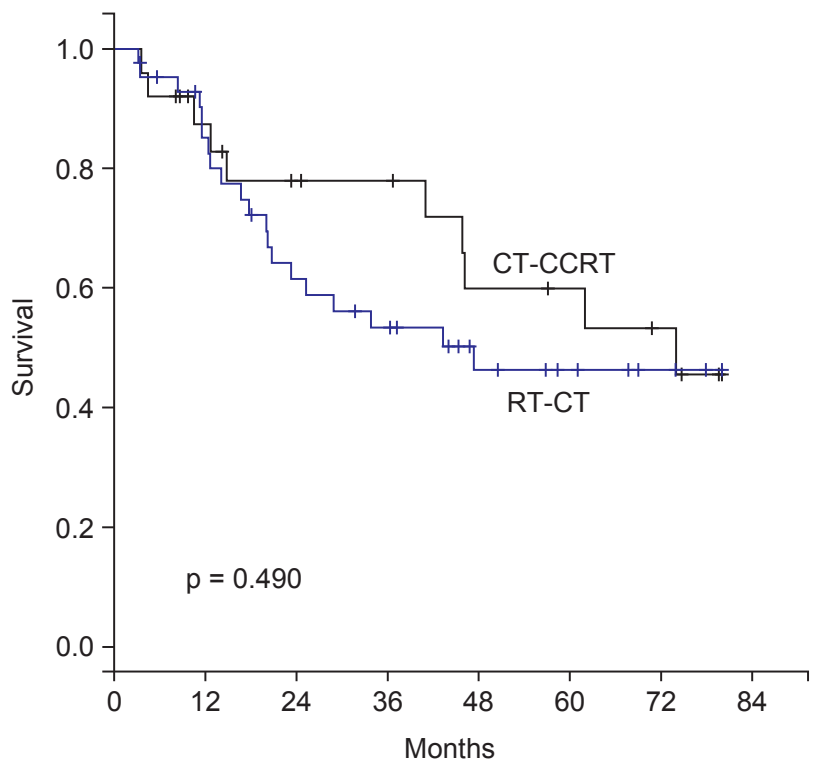

B

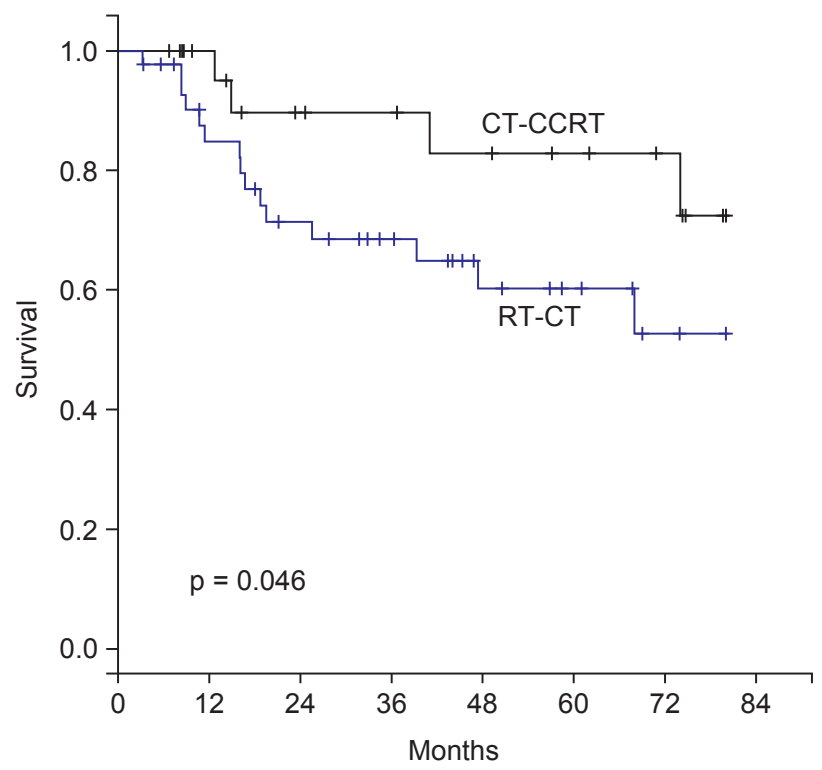

Fig. 1. Kaplan-Meier survival curves. (A) Overall survival. (B) Locoregional recurrence-free survival. (C) Distant metastasisfree survival between adjuvant chemotherapy followed by concomitant chemoradiotherapy (CT-CCRT) and sequential postoperative radiotherapy followed by adjuvant chemotherapy (RT-CT) protocols.

outcome of the RT-CT protocol, but it did not have prognostic values in both univariable and multivariable analyses. The age of patients in the CT-CCRT protocol was older than that of RTCT protocol (mean, 58.7 vs. $52.9 ; p=0.025$ ) and this may have negatively affected the outcomes in patients treated with the CT-CCRT protocol. However, age also was not a prognostic factor for survivals (Tables 3 and 4). Radiation was more irradiated in RT-CT protocol than CT-CCRT protocol, due to the different proportion of RT-techniques used in the protocols. In the clinical setting, the dose difference of $3 \mathrm{~Gy}$ did not seem to be significant (mean, 52.8 Gy vs. $55.8 \mathrm{~Gy} ; \mathrm{p}=0.013$ ).
Our CT-CCRT protocol had been adopted during a time before POCT became a standard of care (from October 1996 to mid-2005). But, the RT-CT protocol, which was established in mid-2005, has been implemented as a current active protocol in our institution. Considering the variety of developments in chemotherapy and radiotherapy over time, the recent RT-CT protocol should have showed superior outcomes compared to that of the old CT-CCRT protocol. However, the two protocols did not show significant differences in OS and DMFS. Rather, the CT-CCRT protocol showed superior outcomes compared to the RT-CT protocol in controlling locoregional disease, despite 
Table 4. Multivariable analyses for clinical variables affecting survivals

\begin{tabular}{lccccccccc}
\hline \multirow{2}{*}{\multicolumn{1}{c}{ Variable }} & \multicolumn{4}{c}{ LRFS } & \multicolumn{3}{c}{ DMFS } & \multicolumn{2}{c}{ OS } \\
\cline { 2 - 10 } & HR & $95 \% \mathrm{Cl}$ & $\mathrm{p}$-value & $\mathrm{HR}$ & $95 \% \mathrm{Cl}$ & $\mathrm{p}$-value & $\mathrm{HR}$ & $95 \% \mathrm{Cl}$ & $\mathrm{p}$-value \\
\hline Age (<58 vs. $\geq 58$ yr) & - & - & - & - & - & - & 1.284 & $0.535-3.082$ & 0.575 \\
Gender (male vs. female) & 2.525 & $0.827-7.712$ & 0.104 & 1.525 & $0.647-3.592$ & 0.334 & - & - & - \\
ECOG PS (0-1 vs. 2) & 0.754 & $0.183-3.100$ & 0.696 & 1.567 & $0.504-4.867$ & 0.430 & - & - & - \\
Comorbidity index (<2 vs. $\geq 2)$ & - & - & - & - & - & - & 1.594 & $0.532-4.772$ & 0.405 \\
Preoperative FEV1 (<2.5 L vs. $\geq 2.5$ L) & 0.581 & $0.195-1.720$ & 0.326 & - & - & - & - & - & - \\
Tumor histology (squamous vs. others) & - & - & - & 0.775 & $0.315-1.909$ & 0.580 & - & - & - \\
Protocol (CT-CCRT vs. RT-CT) & 3.506 & $1.020-12.053$ & 0.046 & - & - & - & 0.647 & $0.318-1.315$ & 0.229 \\
\hline
\end{tabular}

LRFS, locoregional recurrence-free survival; DMFS, distant metastasis-free survival; OS, overall survival; $\mathrm{HR}$, hazard ratio; $\mathrm{Cl}$, confidence interval; ECOG PS, Eastern Cooperative Oncology Group performance status; FEV1, forced expiratory volume in 1 second; CT-CCRT, adjuvant chemotherapy followed by concomitant chemoradiotherapy; RT-CT, sequential postoperative radiotherapy followed by adjuvant chemotherapy.

CCRT protocol having mainly adopted 2D-RT (Fig. 1B) (Table 4). These results suggest that the CT-CCRT protocol can be effective in controlling locoregional disease, which may lead to a potential OS benefit.

Although its use has been an issue of debate, PORT can be administered in patients with N2 disease based on several large-scale population-based studies which favored the use of PORT even in the era of POCT $[5,8,9]$. When PORT is determined to be implemented in the clinical setting, the way of combining PORT with POCT can become an important issue in order to maximize the effect of adjuvant therapies. Currently, POCT followed by PORT is generally accepted when combining two adjuvant therapies due to the concrete evidence supporting the survival benefit of POCT. However, the POCT-first strategy may delay the start of PORT delayed by more than 4-5 months after surgery. Considering the relatively high locoregional tumor burden compared to that of systemic metastasis and the better response rate of radiation therapy than chemotherapy, delaying PORT may lead to the loss of opportune timing in controlling locoregional residual tumors. In the case of PORT-first, POCT can be started within 3 months after surgery due to the shorter treatment time of PORT (5-6 weeks) compared to that of POCT (12-16 weeks). Our RT-CT protocol reflected such rationales, and we reported that the outcomes of PORT-first strategy were comparable to those of the POCT-first strategy, while also preserving the survival benefit of POCT [10]. In this context, concurrent administration of PORT and POCT (CCRT) can be an ideal way to combine two adjuvant therapies. It can minimize the delay time of both PORT and POCT. Locoregional control can be achieved more effectively by radio-sensitization. This benefit, however, can be offset by increases in treatment-related complications.
The results of this study show the improved LRFS of the CTCCRT protocol compared to the RT-CT protocol without any apparent increases in complications. Although these results may not represent all complications, several other studies adopting the CCRT protocol report its comparable outcomes and acceptable complication profiles [15-19]. Shen et al. [20] reported an early closed randomized trial comparing POCT vs. postoperative concurrent chemoradiotherapy (POCRT). This trial showed that POCRT increased both locoregional and distant disease free survival rate compared with POCT alone in patients with IIIA-N2 NSCLC. However, there was no increase of OS rate in PORCT. Although there have been no definite studies supporting the benefit of adjuvant CCRT, the tendency of increased LRFS or DFS suggests the need of a large-scaled randomized trial evaluating the OS benefit comparing CCRT with POCT alone or sequential RT-CT/CT-RT strategies in patients with IIIA-N2 disease.

The result of this study are limited because of the comparison between two protocols were performed consecutively in our institution. This study is also limited by a small sample size, heterogeneous study population (including pN1 disease) and single-institutional retrospective study design. However despite these limitations, the results of this study suggest again the potential role of adjuvant CCRT in locoregional tumor control.

In conclusion, CT-CCRT protocol increased LRFS compared to RT-CT protocol in patients with completely resected NSCLC, but not in OS and DMFS. Further large-scaled randomized studies are warranted to evaluate the benefit of CCRT strategy compared with sequential CT-RT or RT-CT strategy. 


\section{Conflict of Interest}

No potential conflict of interest relevant to this article was reported.

\section{Acknowledgments}

This work was supported by the new faculty research fund of Ajou University School of Medicine. The authors thank Stephen Kim for assistance with manuscript editing.

\section{References}

1. Douillard JY, Rosell R, De Lena M, et al. Adjuvant vinorelbine plus cisplatin versus observation in patients with completely resected stage IB-IIIA non-small-cell lung cancer (Adjuvant Navelbine International Trialist Association [ANITA]): a randomised controlled trial. Lancet Oncol 2006;7:719-27.

2. Pignon JP, Tribodet $H$, Scagliotti GV, et al. Lung adjuvant cisplatin evaluation: a pooled analysis by the LACE Collaborative Group. J Clin Oncol 2008;26:3552-9.

3. Postoperative radiotherapy in non-small-cell lung cancer: systematic review and meta-analysis of individual patient data from nine randomised controlled trials: PORT Metaanalysis Trialists Group. Lancet 1998;352:257-63.

4. Billiet $C$, Decaluwe $H$, Peeters $S$, et al. Modern post-operative radiotherapy for stage III non-small cell lung cancer may improve local control and survival: a meta-analysis. Radiother Oncol 2014;110:3-8.

5. Lally BE, Zelterman D, Colasanto JM, Haffty BG, Detterbeck FC, Wilson LD. Postoperative radiotherapy for stage II or III nonsmall-cell lung cancer using the surveillance, epidemiology, and end results database. J Clin Oncol 2006;24:2998-3006.

6. Douillard JY, Rosell R, De Lena M, et al. Impact of postoperative radiation therapy on survival in patients with complete resection and stage I, II, or IIIA non-small-cell lung cancer treated with adjuvant chemotherapy: the adjuvant Navelbine International Trialist Association (ANITA) Randomized Trial. Int J Radiat Oncol Biol Phys 2008;72:695-701.

7. Corso $C D$, Rutter $C E$, Wilson $L D$, Kim AW, Decker RH, Husain ZA. Re-evaluation of the role of postoperative radiotherapy and the impact of radiation dose for non-small-cell lung cancer using the National Cancer Database. J Thorac Oncol 2015;10:148-55.

8. Mikell JL, Gillespie TW, Hall WA, et al. Postoperative radiotherapy is associated with better survival in non-small cell lung cancer with involved N2 lymph nodes: results of an analysis of the National Cancer Data Base. J Thorac Oncol 2015;10:462-71.

9. Robinson CG, Patel AP, Bradley JD, et al. Postoperative radiotherapy for pathologic N2 non-small-cell lung cancer treated with adjuvant chemotherapy: a review of the National Cancer Data Base. J Clin Oncol 2015;33:870-6.

10. Lee HW, Noh OK, Oh YT, et al. Radiation therapy-first strategy after surgery with or without adjuvant chemotherapy in stage IIIA-N2 non-small cell lung cancer. Int J Radiat Oncol Biol Phys 2016;94:621-7.

11. Jeong SH, Lee HW, Han JH, et al. Low expression of Bax predicts poor prognosis in resected non-small cell lung cancer patients with non-squamous histology. Jpn J Clin Oncol 2008;38:661-9.

12. Sundararajan $V$, Henderson $T$, Perry $C$, Muggivan $A$, Quan $H_{\text {, }}$ Ghali WA. New ICD-10 version of the Charlson comorbidity index predicted in-hospital mortality. J Clin Epidemiol 2004;57:1288-94.

13. $R$ Development Core Team. R: a language and environment for the statistical computing [Internet]. Vienna, Austria: The R Foundation; c2016 [cited 2016 Aug 18]. Available from: https://www.r-project.org/.

14. Kim H, Lussier YA, Noh OK, Li H, Oh YT, Heo J. Prognostic implication of pulmonary function at the beginning of postoperative radiotherapy in non-small cell lung cancer. Radiother Oncol 2014;113:374-8.

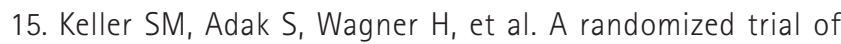
postoperative adjuvant therapy in patients with completely resected stage II or IIIA non-small-cell lung cancer. Eastern Cooperative Oncology Group. N Engl J Med 2000;343:1217-22.

16. Bradley JD, Paulus R, Graham MV, et al. Phase II trial of postoperative adjuvant paclitaxel/carboplatin and thoracic radiotherapy in resected stage II and IIIA non-small-cell lung cancer: promising long-term results of the Radiation Therapy Oncology Group: RTOG 9705. J Clin Oncol 2005;23:3480-7.

17. Wakelee HA, Stephenson P, Keller SM, et al. Post-operative radiotherapy (PORT) or chemoradiotherapy (CPORT) following resection of stages II and IIIA non-small cell lung cancer (NSCLC) does not increase the expected risk of death from intercurrent disease (DID) in Eastern Cooperative Oncology Group (ECOG) trial E3590. Lung Cancer 2005;48:389-97.

18. Feigenberg SJ, Hanlon AL, Langer $C$, et al. A phase II study of concurrent carboplatin and paclitaxel and thoracic radiotherapy for completely resected stage II and IIIA nonsmall cell lung cancer. J Thorac Oncol 2007;2:287-92.

19. Lee HC, Kim YS, Oh SJ, et al. The single institutional outcome of postoperative radiotherapy and concurrent chemoradiotherapy in resected non-small cell lung cancer. Radiat Oncol J 2014;32:147-55.

20. Shen WY, Ji J, Zuo YS, et al. Comparison of efficacy for postoperative chemotherapy and concurrent radiochemotherapy in patients with IIIA-pN2 non-small cell lung cancer: an early closed randomized controlled trial. Radiother Oncol 2014;110:120-5. 